\title{
Leishmaniose visceral e gestação: relato de caso
}

\author{
Visceral leishmaniasis (kala-azar) and pregnancy: a case report
}

Ernesto Antonio Figueiró Filho ${ }^{1}$, Sílvia Naomi Oliveira Uehara ${ }^{1}$, Flávio Renato de Almeida Senefonte ${ }^{1}$, Alessandro Henrique Antunes Lopes ${ }^{1}$, Geraldo Duarte ${ }^{2}$, Patrícia El Beitune ${ }^{2}$

\section{RESUMO}

Leishmaniose visceral (LV) é doença endêmica no Estado de Mato Grosso do Sul, Brasil, principalmente nas áreas próximas dos rios Paraguai e Paraná. Nos últimos anos vem aumentando o número de casos, especialmente na Capital, Campo Grande, com conseqüente surgimento de casos em gestantes e conseqüentemente havendo risco de transmissão vertical do parasita. No presente trabalho é relatado um caso de LV em gestante, acompanhada por nosso grupo e tratada com anfotericina B lipossomal, não tendo ocorrido a transmissão vertical do parasita. O presente relato demonstra opção terapêutica em casos de calazar durante a gestação, tendo em vista a contra-indicação relativa do uso do antimoniato, droga de primeira escolha para tratamento em pacientes não-gestantes.

PALAVRAS-CHAVE: Leishmaniose visceral; Gestação; Infecção tropical; Transmissão vertical

\section{ABSTRACT}

Visceral leishmaniasis (VL) is an endemic disease in the state of Mato Grosso do Sul, Brazil, mainly in the areas near the Paraguay and Paraná rivers. An increasing number of cases have been occurring especially in the state capital Campo Grande, with consequent occurrence of VL cases in pregnant women. This situation causes an elevated risk of vertical transmission of the parasite. In this report, we describe a case of VL in a pregnant woman followed up by our group, who was treated with liposomal amphotericin B, with no vertical transmission of the parasite. In our report, we demonstrate a therapeutic option for kala-azar during pregnancy, since antimoniate, the first-choice drug, is not prescribed during the gestational period.

KEYWORDS: Visceral leishmaniasis; Pregnancy; Tropical infection; Vertical transmission

\section{Introdução}

Leishmaniose visceral (LV) é doença tropical, causada pelo protozoário do gênero Leishmania, cujas espécies ocorrem no Mediterrâneo e na América do Sul. Sendo uma zoonose, a transmissão predominante é vetorial, necessitando do mosquito flebotomíneo, tendo um ciclo biológico com o cão como reservatório $^{1,2}$. Todavia, a transmissão pode ser também transfusional e vertical ${ }^{1-3}$, esta última, por meio da passagem de formas amastigotas pela placenta durante o período gestacional.

Outras formas de transmissão menos freqüentes incluem acidente de laboratório com objetos contaminados e compartilhamento de seringas entre usuários de drogas, sendo esta encontrada como principal forma de transmissão nos casos de LV em pacientes infectados pelo vírus da imunodeficiência humana.

1 Departamento de Ginecologia e Obstetrícia do Centro de Ciências Biológicas da Universidade Federal de Mato Grosso do Sul

2 Departamento de Ginecologia e Obstetrícia da Faculdade de Medicina de Ribeirão Preto da Universidade de São Paulo

Correspondência: Ernesto Antonio Figueiró-Filho

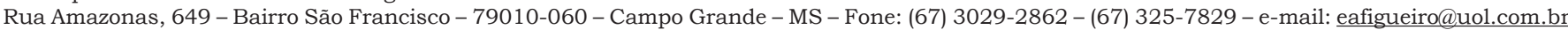

Recebido em: 27/8/2004 Aceito com modificações em: 27/12/2004 
O quadro clínico varia desde formas assintomáticas até formas clássicas de calazar, com evolução crônica podendo causar óbito se não for diagnosticada e corretamente tratada ${ }^{1-4}$. A LV é endêmica no estado de Mato Grosso do Sul, com predomínio antigo nas regiões próximas dos rios Paraguai e Paraná, acometendo o Pantanal sulmatogrossense (Corumbá, Bodoquena, Miranda, Jardim, Bonito, Aquidauana) e Três Lagoas, na divisa com o Estado de São Paulo. Nos últimos anos, porém, vem crescendo o número de casos na capital (Campo Grande), oriundos de áreas urbanas específicas, com alta prevalência de LV em cães. Deste modo, surgem também casos de LV em crianças e mulheres. Nestas, se gestantes, existe o risco de transmissão vertical (TV), acrescido do impacto na morbidade e mortalidade materno-fetal ${ }^{1,3}$.

No Brasil e no mundo, há poucos relatos de LV em gestantes, sendo que, dos disponíveis, a procedência dos casos é de regiões sabidamente endêmicas para a doença. O presente trabalho relata um caso de LV em gestante procedente de Campo Grande-MS.

\section{Relato de Caso}

A paciente, 18 anos, branca, casada, natural e procedente de Campo Grande, foi atendida no Pronto Atendimento do Hospital Regional de Mato Grosso do Sul (HRMS) em 16 de janeiro de 2004 , com idade gestacional de 26 semanas segundo ultra-sonografia obstétrica do primeiro trimestre. Encontrava-se na segunda gestação, com um parto cesáreo anterior há dois anos. A paciente relatou história de dois meses de febre, astenia, cansaço, hiporexia, mialgias e cefaléia. Havia antes procurado várias vezes atendimento médico em unidades básicas de saúde (UBS), sempre recebendo tratamento paliativo e não sendo encaminhada para investigação ambulatorial. No acompanhamento pré-natal, em UBS, não houve investigação para as manifestações clínicas que a paciente estava apresentando, continuando o tratamento paliativo. Negou intercorrências na gestação anterior e abortos.

Estava em regular estado geral, regular estado nutricional, hipocorada $++/ 4$, acianótica, anictérica, sem linfonodos palpáveis, febril $\left(38,7^{\circ} \mathrm{C}\right)$, eupnéica. A ausculta cardiopulmonar não apresentava alterações. O abdome era globoso e a altura uterina de $22 \mathrm{~cm}$. O figado e o baço eram palpáveis a $10 \mathrm{~cm}$ dos respectivos rebordos costais e edema de membros inferiores com cacifo $+/ 4$.
Foram solicitados hemograma, níveis séricos de sódio, potássio, uréia, creatinina, amilase, cálcio e magnésio, exame de urina e urocultura e ultrasonografia obstétrica e de abdome total.

Aos exames de entrada, apresentava 2,3 milhões de eritrócitos/dL, 6,4 g\% de hemoglobina e 600 leucócitos com $60 \%$ de segmentados e $40 \%$ de linfócitos. Havia também plaquetopenia (59.000), caracterizando a pancitopenia. Havia também hipocalemia $(3 \mathrm{mEq} / \mathrm{L})$ e hiponatremia $(126 \mathrm{mEq} / \mathrm{L})$. O exame de urina apresentou-se sem alterações. A urocultura foi negativa. A ultrasonografia abdominal confirmou a hepatoesplenomegalia. A ultra-sonografia obstétrica revelou feto único, vivo, com IG de 26 semanas e índice de líquido amniótico (ILA) de 8,2 cm.

Do acompanhamento pré-natal, possuía exames de tipagem sanguínea $(\mathrm{B} \mathrm{Rh}+$ ) e triagem para sífilis (VDRL), toxoplasmose IgM, herpes simples 1 e $2 \operatorname{IgM}$, virus da imunodeficiência humana 1 e 2, citomegalovírus IgM, hepatite $\mathrm{B}$ e $\mathrm{C}$, vírus linfotrófico humano 1 e 2, rubéola aguda, doença de Chagas e fenilcetonúria materna, todos negativos. A triagem de anticorpos IgG para toxoplasmose, herpes simples, citomegalovirus e rubéola foram positivos.

A paciente foi internada na enfermaria de Ginecologia e Obstetrícia (GO), permanecendo por sete dias, sendo transferida para o CTI no dia 22/ $01 / 04$, por quadro de rebaixamento de consciência, hipofonese de bulhas e piora da pancitopenia, tendo recebido dez unidades de crioprecipitado, permanecendo por três dias em terapia intensiva. Foi submetida a ecocardiografia (ECG), que demonstrou presença de discreto derrame pericárdico, sem restrição diastólica. A paciente também apresentou quadro de otite média aguda supurada, com evolução para otorréia piossanguinolenta, sendo tratada com amoxicilina $500 \mathrm{mg}$ e clavulanato $125 \mathrm{mg}$ via oral $8 / 8 \mathrm{~h}$, sendo então acompanhada pelo serviço de Otorrinolaringologia. Tendo tido alta do CTI, retornou à enfermaria da GO, continuando o tratamento para a otite média aguda até completar 14 dias de antibioticoterapia. Passou a apresentar icterícia 3+/4, estando o baço palpável a $12 \mathrm{~cm}$ do rebordo costal esquerdo e o figado palpável a $10 \mathrm{~cm}$ do rebordo costal direito, confirmados por nova ultrasonografia abdominal.

Após o retorno para a enfermaria de GO, no dia 25/01/04, foi levantada a hipótese de leishmaniose visceral, sendo solicitado aspirado de medula óssea com cultura, encontrando formas amastigotas do parasita no esfregaço a fresco, corado pelo Giemsa. Iniciou-se, então, tratamento imediato para a leishmaniose visceral com 
anfotericina B lipossomal, 50 mg diluídos em 250 $\mathrm{mL}$ de solução glicosada a $5 \%$ intravenosa por duas horas, diariamente, sendo solicitados exames laboratoriais, com periodicidade de três vezes por semana: hemograma, tempo de protrombina, alaninaminotransferase, aspartatoaminotransferase, proteínas totais e frações, creatinina, potássio, ECG e glicemia sérica.

Seis dias após o início do tratamento (31/ $01 / 04)$, a gestante apresentou indícios de sofrimento fetal agudo, com a freqüência cardiaca fetal variando de 85 a 100 batimentos por minuto (bpm), associado a oligoâmnio grave, demonstrado por ultra-sonografia obstétrica, com ILA de $4,8 \mathrm{~cm}$. Nessa ocasião, com 28 semanas de gravidez, foi novamente transferida para cuidados intensivos, com insuficiência hepática e distúrbio da coagulação. Questionou-se a resolução da gestação, postergada devido às condições clínicas desfavoráveis para procedimento cirúrgico e prematuridade do feto. Após três dias de tratamento intensivo (03/ 02/04), mantido o esquema com anfotericina B lipossomal, observou-se estabilização da vitalidade fetal, com melhora da oligodramnia (ILA $=5,5$ $\mathrm{cm})$. A gestante retornou à enfermaria, dia 04/ 02/04, já com dez dias de tratamento para LV, permanecendo internada pelo oligoâmnio, mas com melhora do quadro clínico do calazar manifestado por desaparecimento da febre e regressão da hepatoesplenomegalia, conforme pode ser observado na Tabela 1.

Tabela 1 - Evolução clínica e laboratorial de gestante com leishmaniose visceral durante o tratamento com anfotericina B lipossomal.

\begin{tabular}{|c|c|c|c|c|}
\hline \multirow[t]{2}{*}{ Exame/sinal } & \multirow{2}{*}{$\begin{array}{c}\text { Admissão } \\
16 / 01 / 04\end{array}$} & \multicolumn{2}{|c|}{ Após tratamento } & \multirow{2}{*}{$\begin{array}{c}\text { Alta } \\
3 / 3 / 04\end{array}$} \\
\hline & & 4/2/04 (D10) & 15/02/04 (D21) & \\
\hline \multicolumn{5}{|l|}{ Hemograma: } \\
\hline Eritrócitos $\left(10^{6} / \mathrm{mm}^{3}\right)$ & 2,3 & 2,7 & 6,8 & 4,7 \\
\hline Hemoglobina (g/dL) & 6,4 & 6,6 & 7,3 & 9,1 \\
\hline Hematócrito (\%) & 32 & 35 & 43 & 41 \\
\hline Leucócitos $\left(10^{2} / \mathrm{mm}^{3}\right)$ & 6 & 11 & 78 & 81 \\
\hline Plaquetas $\left(10^{3} / \mathrm{mm}^{3}\right)$ & 59 & 137 & 160 & 185 \\
\hline Albumina (g/dL) & 2,5 & 2,8 & 2,9 & 3,4 \\
\hline Globulina (g/dL) & 4,1 & 4,2 & 3,8 & 3,1 \\
\hline Sódio (mEq/L) & 126 & 135 & 139 & 142 \\
\hline Potássio (mEq/L) & 3 & 4,6 & 5,3 & 5,4 \\
\hline \multicolumn{5}{|c|}{ Ultra-sonografia abdominal } \\
\hline Fígado-RCD (cm) & 10 & 10 & 5 & 3 \\
\hline Baço-RCE (cm) & 10 & 12 & 4 & 1,5 \\
\hline \multicolumn{5}{|l|}{ Ultra-sonografia obstétrica } \\
\hline ILA (cm) & 8,2 & 5,5 & 7,0 & 8,0 \\
\hline
\end{tabular}

Nota: Início do tratamento $\rightarrow 25$ de janeiro de 2004 (D0).

Completaram-se 21 dias de tratamento com anfotericina B lipossomal, com regressão da hepatoesplenomegalia, confirmada por ultra-sonografia abdominal e melhora dos parâmetros laboratoriais, bem como do ILA $(7,0 \mathrm{~cm})$ no dia $15 / 02 / 04$. O tratamento com anfotericina B lipossomal estendeu-se da $27^{\mathrm{a}}$ à $31^{\mathrm{a}}$ semana de gravidez. O último episódio de pico febril ocorreu dez dias após início do tratamento com a anfotericina B lipossomal, no dia 04/ 02/04, com 28 semanas. Após o término do tratamento, optou-se por manutenção da internação até remissão total do quadro de calazar e melhora dos parâmetros laboratoriais, clínicos e obstétricos (ILA).
Neste período, não houve sinais alterados no ECG e reduziu-se a inversão da relação albuminaglobulina significativamente. Recebeu alta hospitalar em 3 de março de 2004, com 32 semanas e 6 dias de gestação, após 48 dias de internação, com orientação de acompanhamento ambulatorial conjunto no serviço de doenças infecto-parasitárias (DIP) e pré-natal no serviço de gestação de alto risco da Universidade Federal de Mato Grosso do Sul (UFMS), ainda com oligoâmnio leve (ILA $=8,0 \mathrm{~cm}$ ). A paciente manteve-se assintomática, seguindo normalmente o pré-natal, sem maiores intercorrências no período. 
Com 38 semanas, a paciente deu entrada na maternidade do HRMS em trabalho de parto, com dilatação de $3 \mathrm{~cm}$, bolsa íntegra, apresentação cefálica e batimentos cardíacos fetais $=148$ $\mathrm{bpm}$. Teve parto normal, sem intercorrências, com RN vivo, do sexo masculino, pesando $2.995 \mathrm{~g}$, tendo $48 \mathrm{~cm}$ de estatura e Apgar de 9 e 10 no $1^{\circ}$ e $5^{\circ}$ minuto pós-parto. Não apresentava malformações e o exame anatomopatológico da placenta não evidenciou o parasita. Em três meses e seis meses com quadro clínico normal, o RN foi submetido a triagem sorológica de sangue venoso periférico, por reação de imunofluorescência indireta (RIFI) IgM e IgG antileishmaniose humana, cujos resultados foram negativos. A puérpera recuperou peso, melhorou da anemia, amamenta ao seio exclusivamente e até novembro de 2004, fazia acompanhamento no Hospital Dia do serviço de DIP da UFMS, sem alterações. A criança, atualmente com 8 meses, encontra-se também em seguimento no serviço de Pediatria da UFMS, sem maiores intercorrências e com desenvolvimento normal para sexo e idade, mantendo sorologias e clínica negativas para leishmaniose visceral.

\section{Discussão}

A Organização Mundial de Saúde estima cerca de 500 mil casos novos de LV por ano no mundo, sendo 2 a 3 mil casos novos anuais somente no Brasil ${ }^{1,2}$. No entanto, não existe estimativa de calazar em gestantes, principalmente em função de poucas publicações de casos, mesmo de áreas endêmicas.

O primeiro relato de LV nas Américas data de 1913, no Paraguai, de paciente proveniente do estado de Mato Grosso ${ }^{1,2}$. Caldas et al. ${ }^{4}$ citam que o primeiro caso relatado de LV em gestante foi de Low e Cooke, em 1926, em gestante africana, com seu recém-nascido apresentando sinais e sintomas de LV, confirmado posteriormente como sendo de TV. Em 1955, outro caso foi relatado na Inglaterra, de gestante que tinha residido na Índia, também com a criança tendo manifestações clínicas e confirmação laboratorial no sétimo mês de vida ${ }^{4-7}$.

Em 1999, Meinecke et al. ${ }^{8}$ chamaram a atenção por relatar um caso de TV de LV, estando a gestante assintomática. Neste caso, a criança passou a apresentar sinais e sintomas compatíveis com o calazar com 16 meses de vida, sendo que nunca tinha estado em região endêmica e não havia vetores onde residia. Contudo, sua mãe esteve em países do Mediterrâneo, endêmicos de LV, sem no entanto ter apresentado manifestações clínicas, todavia apresentava sorologia e teste de Montenegro positivos.

Na América do Sul, a LV em gestantes é considerada rara, devido aos poucos trabalhos disponiveis relatando estes casos, conforme recente revisão sobre calazar e gravidez ${ }^{3}$. No Brasil, principalmente em áreas urbanas e periurbanas, o aparecimento de calazar entre gestantes começa a tornar-se mais freqüente ${ }^{4}$. O primeiro caso foi relatado em $1993^{3,4}$. Seqüencialmente, outros casos foram relatados como Moraes et al., em 1995 citado por Viana et al. ${ }^{9}$.

Recentemente, em 2003, Caldas et al. ${ }^{4}$ relataram mais um caso de LV durante a gravidez. Foram citados dois casos de calazar em gestantes no Estado do Maranhão, cujos sintomas apareceram no segundo mês e no sétimo mês de gestação, com o diagnóstico confirmado pela presença de formas amastigotas no aspirado de medula óssea. O tratamento utilizado foi com antimonial pentavalente $20 \mathrm{mg} / \mathrm{kg} /$ dia por 20 dias e as crianças nascidas, seguidas laboratorialmente por seis meses, tiveram negativos os testes de imunofluorescência indireta ${ }^{9}$.

No caso relatado por Caldas et $\mathrm{al}^{4}{ }^{4}$, a paciente apresentou as manifestações clínicas, sem saber que estava grávida. Fez-se o diagnóstico de gravidez, estando no primeiro trimestre gestacional, com suspeita de LV, confirmada por meio de RIFI, com título de 1:320 e presença de formas amastigotas no aspirado de medula óssea. Foi escolhido o tratamento com anfotericina B $1 \mathrm{mg} /$ $\mathrm{kg} /$ dia por 14 dias, com bom resultado clínico e laboratorial, não sendo observada a TV em seguimento do RN, que tinha quadro clínico normal e sorologia ELISA e RIFI negativos após seis meses de nascimento.

Conforme pode-se observar, o diagnóstico confirmatório de leishmaniose visceral após o quadro clínico característico é a observação de formas amastigotas no aspirado de medula óssea. Para avaliar a TV, recomenda-se exame anatomopatológico da placenta com pesquisa do parasita ${ }^{10}$ e testes de RIFI e/ou ELISA ${ }^{3,4,11}$ durante o primeiro ano de vida, se a criança for assintomática, ou logo no período de acometimento, se o RN for sintomático.

Deve-se verificar sempre a história epidemiológica, à semelhança do caso descrito na Alemanha ${ }^{8}$, onde a gestante assintomática habitava uma região não endêmica para a doença e houve TV, com RN infectado. Após inquérito epidemiológico sobre viagens, descobriu-se que a gestante havia estado em área endêmica do Mediterrâneo no início da gravidez.

Ainda existe discussão quanto à toxicidade 
das drogas utilizadas para o tratamento do calazar durante a gestação, e os medicamentos disponíveis não são considerados seguros para o uso durante a gravidez. Todavia, o risco materno-fetal é elevado nos casos de LV durante a gestação, aumentando a morbimortalidade materna e fetal, sem a instituição de terapêutica adequada ${ }^{3}$. Desse modo, apesar de não haver concordância sobre a segurança dos medicamentos antileishmânia na gravidez, o tratamento de gestantes com LV está indicado, uma vez que o risco de mortalidade materna e fetal em situações de não tratamento específico pode atingir índices próximos a $90 \% \%^{3,5}$.

As principais drogas disponiveis para tratamento de LV são os antimoniais pentavalentes, sendo o principal representante a N-metilglucamina. Como segunda escolha são disponiveis a aminosidina, a pentamidina e o antibiótico anfotericina $\mathrm{B}^{1-4,12,13}$. Os antimoniais pentavalentes atravessam a barreira placentária podendo causar impregnação neural, ocasionando os mais variados graus de retardo mental no $\mathrm{RN}^{3,4,12,13}$. Há um relato de parto pré-termo após uso do antimonial em gestante com $\mathrm{LV}^{14}$.

A segunda escolha no tratamento de LV é a anfotericina B, contudo, também apresenta efeitos tóxicos, sendo necessário acompanhamento apurado. De acordo com os trabalhos disponiveis, a anfotericina B é a droga mais empregada no tratamento de LV no período gestacional. Outra opção é o aminoglicosídeo aminosidina, com monitorização obrigatória da função renal materna ${ }^{3,15}$.

Em função deste temor quanto à toxicidade das drogas disponiveis, Kumar et al. ${ }^{15}$ relataram um caso em que a gestante foi tratada paliativamente com antitérmicos até o parto, pois se encontrava com 36 semanas de gestação no momento do diagnóstico, não havendo TV. Após o parto, iniciou-se tratamento específico com antimonial pentavalente administrado à mãe.
Modificações do esquema terapêutico vêm sendo aplicadas e sugeridas para reduzir e controlar os efeitos tóxicos dos medicamentos. A anfotericina B apresenta altos indices de cura para LV (93-100\%), sem muitas repercussões sobre o feto ${ }^{4}$. Thakur et al. ${ }^{16}$ relataram o tratamento de quatro gestantes com LV utilizando anfotericina $\mathrm{B}, 1 \mathrm{mg} / \mathrm{kg} /$ dia intravenosa, em infusão lenta de duas horas, por 20 dias.

No Brasil, há poucos relatos de tratamento de gestantes com leishmaniose visceral. Silveira et al. ${ }^{14}$ contra-indicam o uso de antimonial em gestantes com LV, considerando ideal o tratamento com aminosidina, $12-16 \mathrm{mg} / \mathrm{kg}$ intramuscular por 15-20 dias, ou anfotericina $\mathrm{B}, 1 \mathrm{mg} / \mathrm{kg} / \mathrm{dia}$ intravenosa por 14 dias.

A anfotericina B lipossomal apresenta menor nefrotoxidade ${ }^{13}$ e pequena taxa de transferência placentária ${ }^{3,7}$, sendo recomendada uma dose de $1-3 \mathrm{mg} / \mathrm{kg} /$ dia, não ultrapassando o limite de $50 \mathrm{mg} /$ dia, via intravenosa, em solução glicosada a $5 \%$ em 2 horas, por periodo entre 14 e 21 $\operatorname{dias}^{3,7,13}$. Alguns autores preconizam período de 10 a 21 dias $^{3,12,13}$. Quanto aos efeitos colaterais, observa-se risco de hipopotassemia, hipomagnesemia, febre e anemia normocítica e hipocrômica, variando de 27 a $33 \%$ dos casos, e cerca de $5 \%$ de risco de cardiotoxicidade ${ }^{12}$. Exige-se, portanto, um controle de ECG semanal, hemograma, dosagem sérica de sódio, potássio, magnésio, cálcio e creatinina três vezes por semana ou conforme necessidade ${ }^{12,13,17}$.

Gradoni et al. ${ }^{7}$ relataram um caso de gestante com LV tratada com anfotericina B lipossomal, $3 \mathrm{mg} / \mathrm{kg}$ por 10 dias, com regressão laboratorial e clínica após 11 dias do tratamento, com aspirado de medula óssea negativo após 21 dias. Os estudos disponiveis com utilização de tratamento em gestantes sintomáticas, portadoras de LV, podem ser observados na Tabela 2 .

Tabela 2 - Relatos anteriores disponíveis com utilização de tratamento em gestantes sintomáticas portadoras de leishmaniose visceral (LV).

\begin{tabular}{|c|c|c|c|c|c|c|c|}
\hline $\begin{array}{l}\text { Número } \\
\text { de casos }\end{array}$ & Ano & Referência & País & Droga utilizada & Dose diária & $\begin{array}{l}\text { Período idade } \\
\text { gestacional }\end{array}$ & LV congênita \\
\hline 5 & 1993 & Thakur et al. ${ }^{16}$ & Índia & Anfotericina B & $1 \mathrm{mg} / \mathrm{kg}$ & $2 / 3^{\circ}$ trimestre & Não \\
\hline 1 & 1995 & Utili et al. ${ }^{17}$ & Itália & Antimonial & $850 \mathrm{mg} \mathrm{Sb}{ }^{5+}$ & $2^{\circ}$ trimestre & Não \\
\hline 1 & 2001 & Kumar et al. ${ }^{15}$ & Índia & Anfotericina B & $1 \mathrm{mg} / \mathrm{kg}$ & $3^{\circ}$ trimestre & Não \\
\hline 1 & 2003 & Silveira et al. ${ }^{14}$ & Brasil & Antimonial & 850 mg Sb ${ }^{5+}$ & $3^{0}$ trimestre & Não \\
\hline 1 & 2003 & Caldas et al. ${ }^{4}$ & Brasil & Anfotericina B & $1 \mathrm{mg} / \mathrm{kg}$ & $1^{0}$ trimestre & Não \\
\hline
\end{tabular}


Em nosso relato, a gestante estava no início do terceiro trimestre da gestação, quando do diagnóstico, sendo tratada com anfotericina $B$ lipossomal $1 \mathrm{mg} / \mathrm{kg} /$ dia, respeitando a dose máxima de $50 \mathrm{mg} /$ dia, em solução glicosada a $5 \%$ endovenosa em 2 horas por 21 dias, com bom resultado clínico e laboratorial, sem intercorrências. A escolha baseou-se nas referências da literatura, que mostram menor toxicidade deste medicamento. A criança obteve sorologia por RIFI IgM e IgG antileishmaniose humana negativa em seis meses de seguimento, sem manifestações clínicas e sem alterações laboratoriais.

Conclui-se que a ocorrência de calazar no período gestacional demonstra perigo real de TV, sendo a maioria dos casos relatados de gestantes sintomáticas não tratadas. A escassez de trabalhos sobre o assunto em regiões endêmicas levanta as hipóteses de subdiagnóstico, não notificação ou não apresentação dos casos à comunidade científica.

Outra observação é a falta de estudos consistentes que definam um padrão para o tratamento de LV em gestantes de forma mais segura e eficaz, uma vez que os fármacos disponiveis não são seguros para tratamento no periodo gestacional. Desse modo, até que estudos mais consistentes definam o melhor regime terapêutico a ser adotado, os riscos materno-fetais de morbimortalidade em gestações associadas à LV exigem o tratamento nesse período. Até o presente, a droga de escolha é a anfotericina $\mathrm{B}$ ou seus derivados, como a anfotericina B lipossomal, a qual no presente caso demonstrou-se eficaz no tratamento materno e na redução da TV da LV.

\section{Referências}

1. Genaro O. Leishmaniose visceral americana. In: Neves DP, Melo AL, Genaro O, Linardi PM, editores. Parasitologia Humana. $10^{a}$ ed. São Paulo: Atheneu; 2000. p. 56-72.

2. Badaró R, Duarte MIS. Leishmaniose visceral. In: Veronesi R, Focaccia R, editores. Veronesi Tratado de Infectologia. $2^{\text {a }}$ ed. São Paulo: Atheneu; 2002. v. 2, p. 1254-79.

3. Figueiró-Filho EA, Duarte G, El-Beitune P, Quintana SM, Maia TL. Visceral leishmaniasis (kala-azar) in pregnancy. Infect Dis Obstet Gynecol. 2004; 12:31-40.
4. Caldas AJ, Costa JM, Gama ME, Ramos EA, Barral A. Visceral leishmaniasis in pregnancy: a case report. Acta Trop. 2003; 88:39-43.

5. Yadav TP, Gupta H, Satteya U, Kumar R, Mittal V. Congenital kala-azar. Ann Trop Med Parasitol. 1989; 83:535-7.

6. Nyakundi PM, Muigai R, Were JB, Oster CN, Gachihi GS, Kirigi G. Congenital visceral leishmaniasis: case report. Trans R Soc Trop Med Hyg. 1988; 82:564.

7. Gradoni L, Gaeta GB, Pellizzer G, Maisto A, Scalone A. Mediterranean visceral leishmaniasis in pregnancy. Scand J Infect Dis. 1994; 26:627-9.

8. Meinecke CK, Schottelius J, Oskan L, Fleisher B. Congenital transmission of visceral leishmaniasis (kala-azar) from an asymptomatic mother to her child. Pediatrics. 1999; 104:e65.

9. Viana GMC, Nascimento MDSB, Viana MGC, Burattini MM. Transmissão congênita do Calazar. Rev Soc Bras Med Trop. 2001; 34 (Suppl 1):247.

10.Eltoum IA, Zijlstra EE, Ali MS, et al. Congenital kalaazar and leishmaniasis in the placenta. Am J Trop Med Hyg. 1992; 46:57-62.

11.Melby PC. Leishmania In: Behrmam, Kliegman RM, Jensom HB, editors. Nelson - Textbook of Pediatrics. $16^{\text {th }}$ ed. Philadelphia: WB Saunders; 2000. p. 1028-30.

12.Tracy JN, Webster LT Jr. Chemotherapy of infection of protoparasits. In: Hardman JG, Limbird LE, Gilman AG, editors. Goodman \& Gilman: the pharmacological basis of therapeutics. $10^{\text {th }}$ ed. New York: McGraw Hill; 2001. p. 823-40.

13.Bennett JE. Antimicrobians: antifungic agents. In: Hardman JG, Limbird LE, Gilman AG, editors. Goodman \& Gilman: the pharmacological basis of therapeutics. 10 $10^{\text {th }}$ ed. New York: McGraw Hill; 2001. p. 971-4.

14. Silveira BP, Araújo Sobrinho J, Leite LF, et al. Parto prematuro após uso de antimonial pentavalente: relato de um caso. Rev Soc Bras Med Trop. 2003; 36:523-5.

15.Kumar A, Mittal M, Prasad S. Treatment of leishmaniasis in pregnancy. Int $\mathrm{J}$ Gynaecol Obstet. 2001; 72:189-90.

16. Thakur CP, Singh RK, Hassan SM, Kumar R, Narain $\mathrm{S}$, Kumar A. Amphotericin B deoxycholate treatment of visceral leishmaniasis with newer modes of administration and precautions: a study of 938 cases. Trans R Soc Trop Med Hyg. 1999; 93:319-23.

17.Utili R, Rambaldi A, Tripodi MF, Andreana A. Visceral leishmaniasis during pregnancy treated with meglumine antimoniate. Infection. 1995; 23:182-3. 